\title{
Fatores associados aos sentimentos decorrentes da perda dentária total e às expectativas de reposição protética em adultos e idosos
}

\section{Factors associated with feelings arising from total tooth loss and expectations of denture replacement in adults and elderly}

\author{
Livia Fernandes Probst ${ }^{1}$, Gláucia Maria Bovi Ambrosano ${ }^{1}$, \\ Karine Laura Cortellazzi ${ }^{1}$, Luciane Miranda Guerra ${ }^{1}$, Margarete Ribeiro-Dasilva², \\ Scott Tomar², Marina Ciarântola1', Isadora Prata Soldi Konkowski', \\ Rosana de Fátima Possobon ${ }^{1}$
}

\begin{abstract}
Resumo
Introdução: A perda dentária é considerada um evento grave na vida dos pacientes e pode levá-los à reclusão social. A reabilitação por meio de próteses pode contribuir para a melhora da autoimagem de edêntulos totais. Objetivo: Este estudo teve como objetivo conhecer os fatores associados aos sentimentos decorrentes da perda de dentes e às expectativas quanto à nova prótese. Métodos: Estudo transversal analítico com 119 pacientes edêntulos totais (faixa etária entre 29 e 87 anos) em tratamento para confecção de prótese em uma clínica-escola. Resultados: Os pacientes que relataram ter perdido seus dentes devido ao medo de submeter-se ao tratamento ou a dificuldades de acesso foram os que mais sofreram com a perda dentária (OR=2,57). Os pacientes mais jovens $(\mathrm{OR}=3,33)$ e do sexo feminino $(\mathrm{OR}=3,89)$ foram aqueles que demonstraram mais expectativas em relação à prótese que estava sendo confeccionada. Conclusão: As mulheres e as pessoas mais jovens são aquelas que manifestaram mais raiva ou tristeza pela perda dentária e que possuíam expectativas estéticas e de socialização em relação à nova prótese.
\end{abstract}

Palavras-chave: perda de dente; sentimentos; prótese dentária; autoimagem.

\begin{abstract}
Introduction: The tooth loss is considered a serious event on the life of patients and it may take them to social withdrawal. The rehabilitation through prosthetics can improve self-image of total edentulous. Objective: This study aimed to assess factors associated with feelings related to tooth loss and the expectations regarding the new prosthesis. Methods: A cross-sectional study with 119 total edentulous patients (age range: 29-87 years) undergoing prostheses treatment in a University Clinic. Results: The patients who reported having lost their teeth due to fear of undergoing treatment or access difficulties were the ones who suffered most from tooth loss $(O R=2.57)$. Younger patients $(O R=3.33)$ and female $(O R=3.89)$ were those that showed more expectations for the prosthesis that was being made. Conclusion: Women and young people are those who express more anger or sadness due to tooth loss and have aesthetic and socialization expectations for the new prosthesis.
\end{abstract}

Keywords: tooth loss; emotions; dental prosthesis; self concept.

\footnotetext{
${ }^{1}$ Departamento de Odontologia Social, Faculdade de odontologia de Piracicaba, Universidade Estadual de Campinas (UNICAMP) - Piracicaba (SP), Brasil. ${ }^{2}$ College of Dentistry, University of Florida - Gainesville (FL), EUA.

Trabalho realizado na Faculdade de Odontologia de Piracicaba (FOP), Universidade Estadual de Campinas (UNICAMP) - Piracicaba (SP), Brasil. Endereço para correspondência: Rosana de Fátima Possobon - CP 52 - CEP: 13414-903 - Piracicaba (SP), Brasil - Email: possobon@fop.unicamp.br Fonte de financiamento: nenhuma.
}

Conflito de interesses: nada a declarar. 


\section{INTRODUÇÃO}

A perda dentária é um evento grave na vida dos pacientes e é considerada uma importante medida da condição de saúde bucal de uma população $0^{1-3}$. A completa ausência de elementos dentários traz consequências deletérias físicas (reabsorção do rebordo residual e diminuição da função mastigatória) e emocionais (diminuição da capacidade social) ${ }^{4}$, podendo levar os indivíduos à reclusão social ${ }^{5}$.

O edentulismo é temática relevante em saúde pública. As circunstâncias sociais dos indivíduos, somadas à prática odontológica hegemônica, ainda influenciam a escolha da extração dos elementos dentários como uma solução para o alívio da dor, especialmente em populações de baixo nível socioeconômico, exercendo importante papel na prevalência dessa condição ${ }^{6,7}$. As perdas dentárias severas (menos de nove dentes permanentes presentes) são consideradas a $36^{\mathrm{a}}$ condição mais prevalente, com uma estimativa global de $2,3 \%$, segundo estudo sobre carga global de doenças realizado no ano de 2010, evidenciando-se, assim, como um problema de saúde pública em todo o mundo 8 .

A reabilitação por meio de próteses pode ter um efeito positivo no comportamento e na autoimagem dos pacientes, pois, ao restabelecer de maneira adequada a estética e a função mastigatória, contribui para uma melhora na interação social dos pacientes ${ }^{8}$. Todavia, o impacto da perda dentária sobre a vida das pessoas não deve ser subestimado, pois, mesmo aceitando bem suas próteses, muitos pacientes podem ter dificuldade em lidar com o edentulismo ${ }^{9} \mathrm{em}$ razão dos sentimentos negativos associados a essa condição, como vergonha e sensação de incompletude ${ }^{10}$.

Os efeitos da perda dentária no estado geral de saúde devem ser, portanto, observados por meio das principais dimensões da saúde: sintomas físicos, capacidade funcional, funcionamento social e percepção de bem-estar ${ }^{4}$. A análise desses efeitos deve considerar também que o comportamento dos indivíduos é condicionado por suas percepções e pela importância dada a elas ${ }^{10}$. Assim, o conhecimento sobre o sentimento do paciente associado à perda dentária, bem como sua expectativa quanto à reposição protética, pode nortear o planejamento dos serviços de saúde nessa área.

Diante do exposto, este estudo teve como objetivo conhecer os fatores associados aos sentimentos decorrentes da perda de dentes e às expectativas quanto à nova prótese.

\section{MÉTODOS}

\section{Aspectos éticos}

Este estudo foi submetido e aprovado pelo Comitê de Ética em Pesquisa de uma instituição de ensino superior (protocolo $n^{\circ} 188 / 2009$ ).

\section{Desenho do estudo e participantes}

Estudo analítico, transversal, de caráter exploratório e metodologia quantitativa, com base em questionário, realizado entre os anos 2011 e 2012 na cidade de Piracicaba, no Estado de São Paulo. Os sujeitos elegíveis para a pesquisa foram os pacientes em tratamento na clínica de graduação de uma instituição de ensino superior dessa cidade.

Os critérios de inclusão foram: edentulismo total; tratamento já iniciado para confecção de prótese total convencional dupla; pacientes que não apresentavam dificuldades de comunicação que os impediam de responder adequadamente o questionário. Foram excluídos da amostra os dados de pacientes que não responderam integralmente as questões.

\section{Amostra e coleta dos dados}

O cálculo inicial da amostra considerou a prevalência de $50 \%$ $(\mathrm{p}=0,50)$ para as respostas do questionário, nível de confiança de $95 \%$ e erro amostral de $5 \%$. Foi considerada a prevalência de $50 \%$ porque é a que proporcionaria o maior erro amostral e, portanto, qualquer outra estimativa de prevalência requereria uma amostra menor para se obter a mesma precisão.

Foi feito ajuste no tamanho da amostra considerando a correção para população finita, chegando-se a um tamanho amostral mínimo de 113 voluntários, o qual proporcionou um poder do teste $(1-\beta)$ de 0,80 , com nível de significância $\alpha$ de 0,05 para um odds ratio (OR) de 2,0 e porcentagem de respostas no grupo não exposto de $50 \%$.

A amostra final consistiu em 119 indivíduos, entre adultos e idosos, recrutados por uma pesquisadora na sala de espera da clínica de graduação, enquanto aguardavam pelo atendimento odontológico. No primeiro contato, foram explicados ao paciente, individualmente e de forma resumida, o objetivo e a metodologia do estudo, convidando cada participante para voluntariamente fazer parte da amostra. Aqueles que concordaram em participar receberam duas cópias do Termo de Consentimento Livre e Esclarecido (TCLE), das quais uma foi devolvida assinada para a pesquisadora. Após a assinatura do TCLE, procedia-se à entrevista.

\section{Instrumento da pesquisa}

Foi utilizado um questionário estruturado contendo questões fechadas e abertas para acessar as informações sobre a perda dental e os sentimentos associados, o uso de próteses e as mudanças em seu cotidiano decorrentes do edentulismo. As questões abordavam a aceitação da perda do dente, os efeitos dessa perda sobre a autoconfiança e a autoimagem do paciente, e as expectativas quanto à prótese total que estava sendo confeccionada. Além dessas questões, foram coletados dados socioeconômicos e demográficos a fim de caracterizar a 
amostra e verificar associação dessas variáveis com os sentimentos relatados pelos pacientes.

O questionário utilizado nesta pesquisa foi elaborado tendo como base os estudos de Fiske et al. ${ }^{11}$ e Davis et al. ${ }^{9}$. Porém sofreu alterações depois da realização de um estudo-piloto com oito pacientes adultos, com objetivo de avaliar a eficiência do questionário para a coleta dos dados desejados. As respostas desses pacientes não foram incluídas nesta pesquisa.

\section{Variáveis analisadas}

A primeira variável dependente analisada foi o sentimento do paciente ao saber que perderia os dentes, categorizado em tristeza/raiva ou alívio/indiferença. Foi verificada associação com sexo (feminino e masculino), motivo da perda dos dentes (doenças bucais ou medo/falta de acesso a tratamento) e idade da perda dos dentes (dicotomizada pela mediana).
A segunda variável dependente foi a expectativa estética e de socialização do paciente quanto à prótese que estava sendo confeccionada, dicotomizada em sim e não. Verificou-se a associação com as seguintes variáveis: idade do paciente na ocasião da perda dos dentes, tempo decorrido entre a perda dental e a coleta dos dados desta pesquisa, tempo de uso de prótese total, número de próteses totais que já possuiu, renda mensal familiar e grau de instrução (todos dicotomizados pela mediana), além de sexo (feminino e masculino), motivo da perda dos dentes (doenças bucais ou medo/falta de acesso a tratamento), motivo da confecção da nova prótese (primeira prótese total ou defeito na prótese anterior) e fonte de renda (aposentadoria ou outras fontes, que englobavam emprego formal ou informal ou dependência de outros familiares).

Após a análise descritiva (Tabela 1), foram realizadas análises simples e múltiplas, estimando-se os OR brutos e ajustados com os respectivos intervalos de confiança de 95\%. Nas regressões

Tabela 1. Análise descritiva da amostra e sentimentos expressos pelos pacientes com relação à perda dentária

\begin{tabular}{|c|c|c|}
\hline Variável & Categorias & $\mathrm{N}=119$ \\
\hline \multirow{2}{*}{ Idade } & $\leq 64$ anos & $64(54 \%)$ \\
\hline & $>64$ anos & $55(46 \%)$ \\
\hline \multirow{2}{*}{ Sexo } & Feminino & $83(70 \%)$ \\
\hline & Masculino & $36(30 \%)$ \\
\hline \multirow{2}{*}{ Motivo da perda dos dentes } & Doenças bucais & $69(58 \%)$ \\
\hline & Medo ou falta de acesso & $50(42 \%)$ \\
\hline \multirow{2}{*}{ Sentimento ao saber que perderia os dentes } & Tristeza ou raiva & $70(59 \%)$ \\
\hline & Alívio ou indiferença & $49(41 \%)$ \\
\hline \multirow{2}{*}{ Foi difícil aceitar a perda dos dentes } & Sim & $80(67 \%)$ \\
\hline & Não & $39(33 \%)$ \\
\hline \multirow{2}{*}{ Conseguiu aceitar a perda dentária } & Sim & $88(74 \%)$ \\
\hline & Não & $31(26 \%)$ \\
\hline \multirow{3}{*}{ Tempo que levou para aceitar a perda dentária } & $<6$ meses & $64(54 \%)$ \\
\hline & $\geq 6$ meses & $24(20 \%)$ \\
\hline & Não aceitaram a perda & $31(26 \%)$ \\
\hline \multirow{2}{*}{ Estava preparado para as consequências do edentulismo } & Sim & $59(49,5 \%)$ \\
\hline & Não & $60(50,5 \%)$ \\
\hline \multirow{2}{*}{$\begin{array}{l}\text { Alguma ajuda teria feito diferença para lidar com o } \\
\text { edentulismo }\end{array}$} & Sim & $94(79 \%)$ \\
\hline & Não & $25(21 \%)$ \\
\hline \multirow{3}{*}{ Qual ajuda teria feito diferença } & Informativo ou experiência de outro paciente & $27(23 \%)$ \\
\hline & Orientações do cirurgião-dentista & $67(56 \%)$ \\
\hline & Nenhuma ajuda & $25(21 \%)$ \\
\hline \multirow{2}{*}{ Sua alimentação foi prejudicada pelo edentulismo } & Sim & $79(66 \%)$ \\
\hline & Não & $40(34 \%)$ \\
\hline \multirow{2}{*}{ Sua vida social foi prejudicada pelo edentulismo } & Sim & $40(34 \%)$ \\
\hline & Não & $79(66 \%)$ \\
\hline \multirow{2}{*}{ Aspecto pessoal prejudicado pelo edentulismo } & Autoestima ou autoconfiança & $70(59 \%)$ \\
\hline & Nenhum & $49(41 \%)$ \\
\hline \multirow{2}{*}{ Renda familiar } & $\leq \mathrm{R} \$ 1.240,00$ & $66(55 \%)$ \\
\hline & $>\mathrm{R} \$ 1.240,00$ & $53(45 \%)$ \\
\hline \multirow{2}{*}{ Fonte de Renda } & Aposentadoria & $84(71 \%)$ \\
\hline & Outros & $35(29 \%)$ \\
\hline \multirow{2}{*}{ Grau de instrução } & $4^{\mathrm{a}}$ série completa & $82(69 \%)$ \\
\hline & Mais do que $4^{\mathrm{a}}$ série & $37(31 \%)$ \\
\hline \multirow{2}{*}{ Motivo da confecção da prótese total } & Primeira prótese total & $14(12 \%)$ \\
\hline & Defeito na prótese anterior & $105(88 \%)$ \\
\hline
\end{tabular}


logísticas múltiplas, foram testadas as variáveis que apresentaram $\mathrm{p} \leq 0,20$ nas análises simples, permanecendo no modelo aquelas com $\mathrm{p} \leq 0,05$. Os ajustes dos modelos foram avaliados pelo “-2 Log Likelihood" e pelo Critério de Informação de Akaike (AIC). Foram consideradas como variáveis dependentes nos modelos as questões "Qual o sentimento ao saber que perderia os dentes?" " "Você tem expectativas estéticas e de socialização para a nova prótese total?".

\section{RESULTADOS}

A Tabela 1 apresenta a caracterização da amostra final do estudo, cuja soma foi de 119 pacientes, dos quais $70 \%$ eram do sexo feminino. A faixa etária variou entre 29 e 87 anos, com a mediana em 64 anos. Mais da metade (55\%) dos participantes entrevistados tinha renda inferior ou igual a dois salários mínimos vigentes na época da coleta dos dados, com a aposentadoria sendo a principal fonte de renda (71\%). O nível de escolaridade para $69 \%$ da amostra era relativo ao antigo ensino primário (até quatro anos de estudo).

Para aceitar a perda de todos os seus dentes, $54 \%$ dos pacientes afirmaram ter levado um tempo inferior a seis meses, enquanto que 56\% afirmaram que "uma explicação do cirurgião-dentista" teria ajudado a lidar com as consequências da perda dental.

Um pouco mais da metade da amostra (50,5\%) perdeu todos os dentes antes de completar 31 anos de idade e $53 \%$ já eram edêntulos havia pelo menos 30 anos. Tristeza e raiva foram os sentimentos que $59 \%$ dos pacientes tiveram ao saber que perderiam os dentes e $67 \%$ afirmaram ter sido difícil aceitar essa perda.

Muitos pacientes (66\%) relataram dificuldades para alimentar-se após a perda dental. Cerca de $69 \%$ tiveram sua autoimagem e/ou autoconfiança afetadas, enquanto $34 \%$ alegaram prejuízo na vida social devido ao edentulismo.

Mais da metade dos pacientes (58\%) atribuiu a causa do edentulismo às doenças bucais e $42 \%$ relataram que o medo de submeter-se ao tratamento odontológico e/ou dificuldades de acesso a tratamento preventivo e restaurador foram responsáveis pela perda dental.

O principal motivo apresentado para procura pelo tratamento atual (confecção de prótese total dupla) foi "defeito na prótese anterior" (88\%). Além disso, $44 \%$ dos pacientes já possuíram mais de duas próteses.

\section{Sentimentos ao saber que perderia todos os dentes}

Na Tabela 2, que apresenta os dados da associação entre o sentimento do paciente ao saber que perderia os dentes e as variáveis sexo, motivo referido como causa do edentulismo e idade em que ocorreu a perda dental, é possível verificar que as mulheres expressaram mais tristeza e raiva do que os homens $(\mathrm{p}=0,0530)$ e que o medo e as dificuldades de acesso geraram mais tristeza e raiva nos pacientes $(\mathrm{p}=0,0191)$.

\section{Expectativas do paciente quanto à prótese}

A Tabela 3 apresenta os fatores associados com as expectativas estéticas e de socialização para nova prótese. Pacientes mais jovens ( $\mathrm{p}=0,0063)$ e do sexo feminino $(\mathrm{p}=0,0018)$ foram aqueles com maiores expectativas em relação à prótese que estava sendo confeccionada.

\section{DISCUSSÃO}

O presente estudo apresentou um padrão de amostra comum para pacientes em tratamento protético: mulheres idosas, com baixo nível socioeconômico e pouca formação acadêmica ${ }^{3,12}$. As mulheres e os mais jovens foram os indivíduos que expressaram sentimentos mais negativos relacionados à perda dental, quando comparados com os homens e os participantes mais velhos do estudo. Esses achados podem ser reforçados por outro estudo que apontou que as mulheres edêntulas sentiam-se menos confiantes e mais constrangidas, pois acreditavam que o edentulismo as deixava menos atraentes ${ }^{13}$, evidenciando que

Tabela 2. Análise de regressão logística múltipla entre o sentimento ao saber que perderia os dentes e variáveis independentes de pacientes em tratamento para prótese total na clínica de graduação da Faculdade de Odontologia de Piracicaba da Universidade Estadual de Campinas (FOP-UNICAMP)

\begin{tabular}{|c|c|c|c|c|c|c|c|c|c|c|}
\hline \multirow[b]{2}{*}{ Variável } & \multirow[b]{2}{*}{ Categorias } & \multirow[b]{2}{*}{$N=119$} & \multicolumn{2}{|c|}{$\begin{array}{c}\text { Qual seu sentimento ao saber } \\
\text { que perderia os dentes? }\end{array}$} & \multirow[b]{2}{*}{ OR } & \multirow[b]{2}{*}{ IC (95\%) } & \multirow[b]{2}{*}{$\mathbf{p}$} & \multirow[b]{2}{*}{$\begin{array}{c}\text { OR } \\
\text { ajustado }\end{array}$} & \multirow[b]{2}{*}{ IC (95\%) } & \multirow[b]{2}{*}{$\mathbf{p}$} \\
\hline & & & Tristeza/raiva & $\begin{array}{c}\text { Alívio/ } \\
\text { indiferença }\end{array}$ & & & & & & \\
\hline \multirow{2}{*}{ Sexo } & Feminino & $83(70 \%)$ & $54(65 \%)$ & $29(35 \%)$ & 2,3276 & \multirow{2}{*}{$1,0486-5,1665$} & \multirow{2}{*}{0,0579} & \multirow{2}{*}{2,24} & \multirow{2}{*}{ 0,99-5,08 } & \multirow{2}{*}{0,0530} \\
\hline & Masculino & $36(30 \%)$ & $16(44 \%)$ & $20(56 \%)$ & 1,00 & & & & & \\
\hline \multirow{2}{*}{$\begin{array}{l}\text { Motivo } \\
\text { da perda } \\
\text { dos dentes }\end{array}$} & $\begin{array}{l}\text { Doenças } \\
\text { bucais }\end{array}$ & $69(58 \%)$ & $34(49 \%)$ & $35(51 \%)$ & 1,00 & \multirow[b]{2}{*}{$1,22-5,76$} & \multirow[b]{2}{*}{0,0216} & \multirow[b]{2}{*}{2,57} & \multirow[b]{2}{*}{$1,17-5,66$} & \multirow[b]{2}{*}{0,0191} \\
\hline & $\begin{array}{l}\text { Medo/falta } \\
\text { de acesso }\end{array}$ & $50(42 \%)$ & $36(72 \%)$ & $14(28 \%)$ & 2,65 & & & & & \\
\hline \multirow{2}{*}{$\begin{array}{l}\text { Idade } \\
\text { de perda } \\
\text { dos dentes }\end{array}$} & $\leq 31$ anos & $60(50,5)$ & $35(58 \%)$ & $25(42 \%)$ & 1,00 & \multirow[b]{2}{*}{$0,5019-2,1621$} & \multirow[b]{2}{*}{0,9389} & & & \\
\hline & $>31$ anos & $59(49,5 \%)$ & $35(59 \%)$ & $24(41 \%)$ & 1,0417 & & & & & \\
\hline
\end{tabular}


Tabela 3. Associação entre a resposta do paciente quanto às expectativas estéticas e de socialização para nova prótese total e variáveis independentes de pacientes em tratamento para prótese total na clínica de graduação da Faculdade de Odontologia de Piracicaba da Universidade Estadual de Campinas (FOP-UNICAMP)

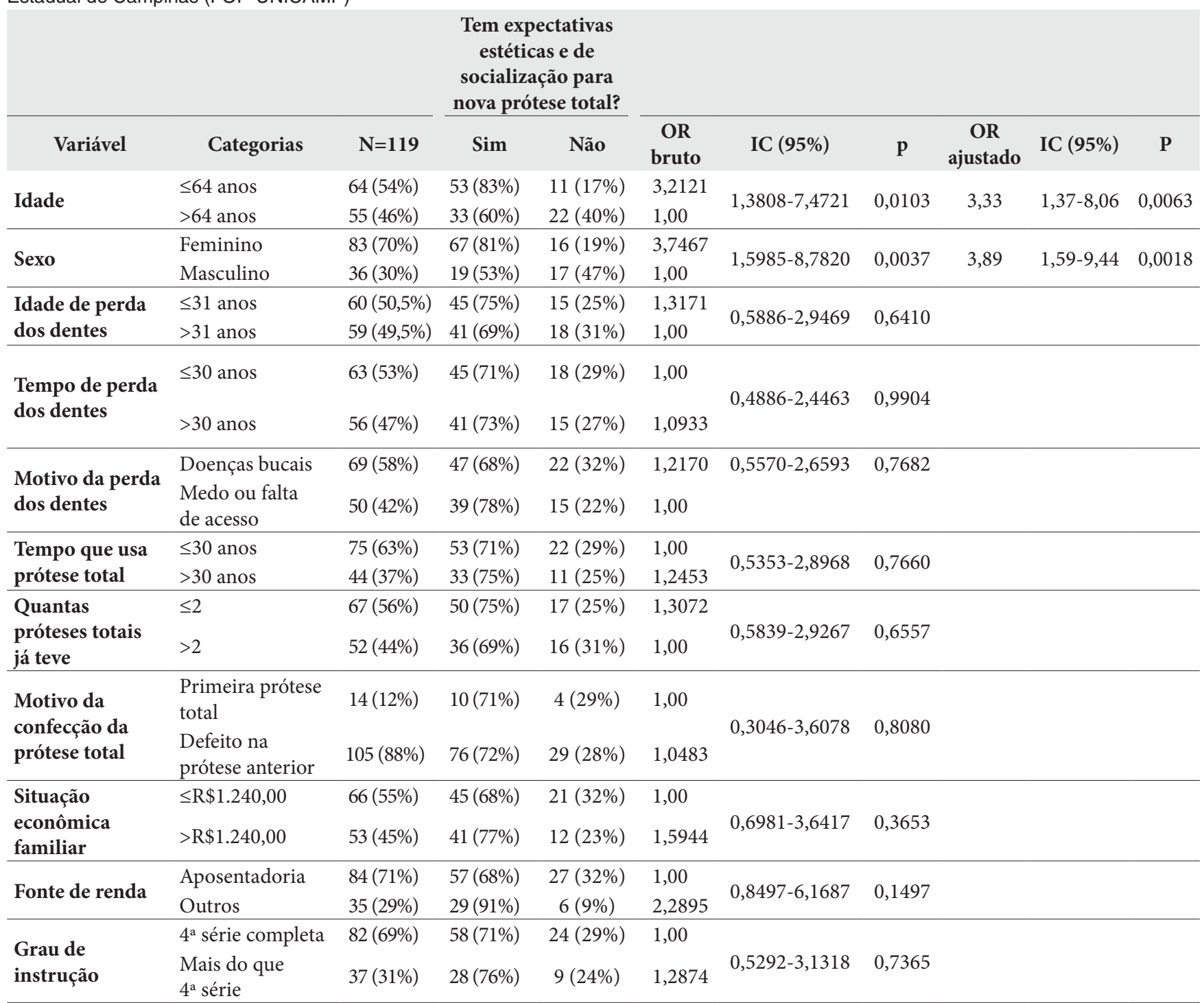

a perda dos dentes causa mais impacto na vida das mulheres do que na dos homens.

As doenças bucais foram apontadas como a principal causa do edentulismo pelos pacientes deste estudo, o que já era esperado, visto que o Brasil possui um longo histórico de baixa qualidade de saúde oral, apesar da grande qualidade técnica e educacional e do grande número de faculdades de odontologia ${ }^{14}$.

Quase metade dos pacientes entrevistados neste estudo era edêntula havia mais de 30 anos, tendo perdido seus dentes por volta das décadas de 1970 e 1980, quando políticas públicas voltadas para a prevenção de problemas bucais ainda eram escassas e direcionadas apenas a certos grupos. Recentemente foram criadas novas políticas no país, como a Estratégia Saúde da Família, implantada em 1994 e proposta para gerar novas ações e cuidados primários de saúde. Embora o acesso ao sistema de saúde seja universal e para todas as idades, historicamente os pacientes mais idosos, como é o caso dos indivíduos deste estudo, não tiveram oportunidade de participar dessas atividades preventivas $^{15}$. Os benefícios de ações de promoção da saúde em grupos etários mais jovens serão observados somente em tempos futuros. Contudo, o momento atual requer iniciativas públicas direcionadas aos idosos de hoje, integrando a agenda de atenção à saúde bucal e atendendo às suas necessidades de tratamento restaurador e reabilitador ${ }^{2}$.

É importante ressaltar que boa parte dos entrevistados atribuiu o edentulismo à dificuldade de acesso ao tratamento ou ao medo de submeter-se ao tratamento odontológico, e não às doenças bucais, evidenciando que esses pacientes acreditam que, com as condições necessárias, poderiam ter mantido seus elementos naturais. Esse quadro reflete duas questões relevantes: (1) ainda existem restrições ao acesso e ao uso de serviços odontológicos especializados no âmbito do Sistema Único de Saúde, em especial 
para a realização de tratamentos endodônticos, fazendo com que a extração se torne um tratamento inevitável quando a cárie se encontra em estágio avançado, com grande destruição tecidual, mormente para os grupos de menor renda ${ }^{12}$; (2) o papel do cirurgião-dentista muitas vezes está associado a práticas mutiladoras representadas pelas exodontias. Consequentemente, as pessoas trazem consigo uma carga de medo e ansiedade muito grande quando buscam por cuidados odontológicos ${ }^{16}$.

O medo, a dor e a ansiedade estão fortemente associados à imagem do dentista ${ }^{17}$ e existem de forma concreta na população brasileira. $\mathrm{O}$ gênero (feminino), a idade (superior a 20 anos) e a experiência de odontalgia são fatores que podem aumentar o grau de ansiedade, evidenciando que há outras variáveis significantes para a manutenção dos dentes que vão além da falta dos recursos econômicos e do descaso com a saúde bucal ${ }^{18}$.

A etiologia do medo e da ansiedade diante do tratamento dental é complexa e multifatorial. Todavia, existe uma implicação prática pertinente indicada pela pesquisa existente nessa área: uma melhor compreensão do medo e da ansiedade do paciente pode evitar a evasão ao tratamento. A fuga do atendimento odontológico impede o paciente de receber informações sobre cuidados preventivos, resultando em má saúde bucal ${ }^{19}$. Essa situação é agravada por sentimentos de constrangimento e vergonha, bem como pela possibilidade de o paciente, que protelou o seu tratamento por longo período, precisar de tratamento mais invasivo, situação com potencial para reforçar ainda mais o medo e a ansiedade, criando um ciclo vicioso que resulta, inevitavelmente, em mutilação dentária ${ }^{20}$.

O papel informativo do cirurgião-dentista teve um destaque entre as ações que poderiam ter minimizado o impacto da perda dentária. Uma explicação do profissional foi considerada muito importante para lidar com as consequências do edentulismo. Cruz et al..$^{17}$ encontraram, em sua pesquisa, que, para os pacientes, o cirurgião-dentista ideal deve conversar e explicar o que vai ser feito por meio de uma linguagem acessível. Por sua vez, as qualidades profissionais técnicas são menos valorizadas. Além disso, os pacientes, principalmente as mulheres, dão ênfase ao diálogo entre profissional e paciente. Essa conversa é considerada importante na medida em que descontrai e tranquiliza o paciente. Avaliar o impacto psicológico do tratamento reabilitador protético é de alta relevância para os profissionais que visam promover a satisfação do paciente. A comunicação do dentista durante o processo de tratamento não pode ser considerada como um processo unidirecional, mas tem de ser interpretada como bidirecional, decisivamente influenciada por um conjunto distinto de características do paciente ${ }^{21}$.

A extração total dos dentes representa uma solução definitiva para a questão da dor, levando muitos pacientes a sentirem-se aliviados quando seus dentes foram extraídos ${ }^{22}$. Apesar de os entrevistados neste estudo terem afirmado sentir raiva ou tristeza diante da perda dental, o percentual daqueles que afirmaram ter sentido indiferença ou alívio foi alto. Na verdade, o desdentado não é considerado doente, e a perda dentária é tratada diferentemente de perdas de outras estruturas do corpo ${ }^{11,13}$. Desmistificar esse fato é essencial para que o profissional possa fornecer um bom suporte aos pacientes ${ }^{15}$. O entendimento de que a remoção dos dentes pode ser a solução para os problemas de saúde bucal é resultado também da articulação de conceitos culturais e médicos ${ }^{13,15}$, o que pode explicar a facilidade com que os entrevistados aceitaram a perda de seus dentes. Em outras palavras, os pacientes conformam-se com o edentulismo apesar de sentirem-se constrangidos devido à perda dentária ${ }^{15}$.

A perda total de dentes representa, antes de tudo, dificuldades para mastigação e alimentação, aspecto igualmente destacado em várias outras pesquisas sobre o tema ${ }^{1,15,23-26}$. Grande parte dos entrevistados relatou ter dificuldades para alimentar-se após o edentulismo; e mais, esperavam que, com a confecção de uma nova prótese, pudessem ser capazes de se alimentar melhor. Além de dificuldades funcionais, ser edêntulo representa também um grande problema estético. A sociedade atual valoriza muito a aparência, e o rosto é a parte mais diferenciada do corpo e está indissociavelmente ligado ao indivíduo e à sua identidade. Os dentes, sem os quais a função do sorriso não se completa devidamente, relacionam-se, mais frequentemente, à juventude, à beleza, à produtividade e à possibilidade de realização. Através da face, uma região sempre exposta do corpo humano, a estética bucal comprometida pode se tornar um motivo de ansiedade ${ }^{27}$.

De fato, um percentual alto dos pacientes entrevistados neste estudo esperava que a prótese que estava sendo confeccionada os deixasse mais bonitos e melhorasse seu convívio social. Consequentemente, os dentes são considerados aspectos importantes nas relações sociais, além de influenciar na autoestima $^{27}$, o que pode explicar o motivo pelo qual $70 \%$ dos entrevistados afirmaram ter sentido sua autoconfiança e autoimagem afetadas pela perda dentária. Em outro estudo que abordou o tema em sauditas, os pacientes atribuíram as alterações na aparência ao envelhecimento, e não à perda dental, e essas alterações foram consideradas inevitávei ${ }^{22}$, fortalecendo a ideia de que a importância dada à aparência varia entre as culturas e de que pacientes do Ocidente, como no caso do presente estudo, tendem a valorizar mais essa questão em suas vidas.

Diversos fatores podem contribuir para um melhor estado de saúde e para a forma como este é percebido, assim como para o bem-estar e para um sentimento de maior felicidade. Os impactos do edentulismo variam de indivíduo para indivíduo ${ }^{13}$, mas uma autoimagem positiva é fator fortemente associada à autopercepção da saúde bucal entre idosos ${ }^{28}$. Isso evidencia a necessidade de um cuidado especial da parte do cirurgião-dentista quando atende a pacientes edêntulos, para que estes se sintam mais preparados para lidar com as consequências da perda dental 
e para que o tratamento reabilitador possa, de fato, responder às suas expectativas.

Ademais, a reabilitação por meio de prótese convencional dupla é ainda uma opção de tratamento devido a algumas limitações da reabilitação por meio de implantes ${ }^{29}$. O impacto psicológico desse tratamento é fortemente influenciado pelo tipo de limitação no bem-estar ocorrido como consequência do edentulismo ${ }^{21}$. Portanto, a satisfação do paciente com essa opção de tratamento é fator de suma importância durante seu planejamento e execução ${ }^{29}$, pois mesmo pacientes que aparentemente conformaram-se bem com o edentulismo tendem a criar expectativas altas para sua prótese $\mathrm{e}^{22}$.

O presente estudo, portanto, contribui com a prática clínica na medida em que disponibiliza informações importantes ao profissional, relativas aos sentimentos e expectativas do paciente. Sugere-se, como continuidade do presente estudo, o seu aprofundamento por meio de abordagens qualitativas, as quais possam melhor elucidar significados e percepções dos sujeitos.

\section{CONCLUSÕES}

As mulheres e as pessoas mais jovens são aquelas que manifestaram mais raiva ou tristeza pela perda dentária e que possuíam expectativas estéticas e de socialização em relação à nova prótese.

Apesar de não ter sido estatisticamente significativo, a importância do cirurgião-dentista no enfrentamento dessa condição também foi ressaltada pelos pacientes entrevistados, que atribuíram a ele as funções de instruir e esclarecer sobre as doenças que levam à perda dentária, suas consequências e opções de tratamento.

\section{REFERÊNCIAS}

1. Vargas AMD, Paixão HH. Perda dentária e seu significado na qualidade de vida de adultos usuários de serviço público de saúde bucal do Centro de Saúde Boa Vista, em Belo Horizonte. Cien Saude Colet. 2005;10(4):1015-24. http://dx.doi.org/10.1590/S1413-81232005000400024.

2. Moreira RS, Nico LS, Tomita NE. O risco espacial e fatores associados ao edentulismo em idosos em município do Sudeste do Brasil. Cad Saude Publica. 2011;27(10):2041-54. PMid:22031208.

3. Papadaki E, Anastassiadou V. Elderly complete denture wearers: a social approach to tooth loss. Gerodontology. 2012;29(2):721-7. PMid:21916954. http://dx.doi.org/10.1111/j.1741-2358.2011.00550.x.

4. Emami E, Souza RF, Kabawat M, Feine JS. The impact of edentulism on oral and general health. International Journal of Dentistry. 2013;498305:7. http:// dx.doi.org/10.1155/2013/498305.

5. Newton JT, Fiske J, Foot O, Frances C, Loh I, Radford DR. A preliminary study of the impact of loss of part of the face and its prosthetic restoration. J Pros Dente. 1999;82:585-590.

6. Mendonça TC. Mutilação dentária: concepções de trabalhadores rurais sobre a responsabilidade pela perda dentária. Cad Saude Publica. 2001;17(6):15457. PMid:11784917. http://dx.doi.org/10.1590/S0102-311X2001000600040.

7. Silva DD, Rihs LB, Sousa MLR. Fatores associados à presença de dentes em adultos de São Paulo, Brasil. Cad Saude Publica. 2009;25(11):2407-18. PMid:19936479. http://dx.doi.org/10.1590/S0102-311X2009001100011.

8. Marcenes W, Kassebaum NJ, Bernabé E, Flaxman A, Naghavi M, Lopez A, et al. Global burden of oral conditions in 1990-2010: a systematic analysis. J Dent Res. 2013;92(7):592-7. PMid:23720570. http://dx.doi. org/10.1177/0022034513490168.

9. Davis DM, Fiske J, Scott B, Radford DR. The emotional effects of tooth loss: a preliminary quantitative study. Br Dent J. 2000;188(9):503-6. PMid:10859849.

10. Haikal DS, Paula AMB, Martins AMEBL, Moreira AN, Ferreira EF. Autopercepção da saúde bucal e impacto na qualidade de vida do idoso: uma abordagem quanti-qualitativa. Cien Saude Colet. 2011;16(7):3317-29. PMid:21808919. http://dx.doi.org/10.1590/S1413-81232011000800031.
11. Fiske J, Davis DM, Frances C, Gelbier S. The Emotional Effects of tooth loss in edentulous people. Br Dent J. 1998;184(2):90-3. PMid:9489217. http:// dx.doi.org/10.1038/sj.bdj.4809551.

12. Peres MA, Barbato PR, Reis SCGB, Freitas CHSM, Antunes JLF. Perdas dentárias no Brasil: análise da Pesquisa Nacional de Saúde Bucal 2010. Rev Saude Publica. 2013;47(Supl 3):78-89. PMid:24626584. http://dx.doi. org/10.1590/S0034-8910.2013047004226.

13. Dable RA, Yashwante BJ, Marathe SS, Gaikwad BS, Patil PB, Momin AA. Toth loss: how emotional it $i$ for the Elderly in India? OHDM. 2014;13(2):305-10. PMid:24984638.

14. Bortoluzzi MC, Traebert J, Lasta R, Rosa TN, Capella DL, Presta AA. Tooth loss, chewing ability and quality of life. Contemp Clin Dent. 2012;3(4):393-7. PMid:23633796. http://dx.doi.org/10.4103/0976-237X.107424.

15. Silva MES, Magalhães CS, Ferreira EF. Perda dentária e expectativa da reposição protética: estudo qualitativo. Cien Saude Colet. 2010;15(3):813-20. PMid:20464194. http://dx.doi.org/10.1590/S1413-81232010000300024.

16. Hiramatsu DA, Tomita NE, Franco LJ. Perda dentária e a imagem do cirurgiãodentista entre um grupo de idosos. Cien Saude Colet. 2007;12(4):1051-6. PMid:17680163. http://dx.doi.org/10.1590/S1413-81232007000400026.

17. Cruz JS, Cota LOM, Paixão HH, Pordeus IA. A imagem do cirurgiãodentista: um estudo de representação social. Rev Odontol Univ Sao Paulo. 1997;11(4):307-13. http://dx.doi.org/10.1590/S0103-06631997000400013.

18. Carvalho RWFD, Falcão PGCB, Campos GJDL, Bastos ADS, Pereira JC Pereira MADS, et al. Ansiedade frente ao tratamento odontológico: prevalência e fatores predictores em brasileiros. Cien Saude Colet. 2012;17(1):1915-22. PMid:22872354. http://dx.doi.org/10.1590/S1413-81232012000700031.

19. Possobon RF, Carrascoza KC, Moraes ABA, Costa Jr ALC. O tratamento odontológico como gerador de ansiedade. Psicol Estud. 2007;12(3):609-16. http://dx.doi.org/10.1590/S1413-73722007000300018.

20. Beaton L, Freeman R, Humphris G. Why are people afraid of the dentist observations and explanations. Med Princ Pract. 2013;23(4):295-301. PMid:24356305. http://dx.doi.org/10.1159/000357223. 
21. Omar R, Tashkandi E, Abduljabbar T, Abdullah MA, Akeel RF. Sentiments expressed in relation to tooth loss: a qualitative study among edentulous saudis. Int J Prosthodont. 2003;16(5):515-20. PMid:14651237.

22. Appollonio I, Carabellese C, Frattola A, Trabucchi M. Dental status, quality of life, and mortality in an older community population: a multivariate approach. J Am Geriatr Soc. 1997;45(11):1315-23. PMid:9361656. http:// dx.doi.org/10.1111/j.1532-5415.1997.tb02930.x.

23. Colussi CF, Freitas SFT. Aspectos epidemiológicos da saúde bucal do idoso no Brasil. Cad Saude Publica. 2002;18(5):1313-20. PMid:12244364. http:// dx.doi.org/10.1590/S0102-311X2002000500024.

24. Colussi CF, Freitas SFT, Calvo MCM. Perfil epidemiológico da cárie e do uso e necessidade de prótese na população idosa de Biguaçu, Santa Catarina. Rev Bras Epidemiol. 2004;7(1):88-97. http://dx.doi.org/10.1590/ S1415-790X2004000100011.

25. Ferreira AAA, Piuvezam G, Werner CWDA, Alves MSCF. A dor e a perda dentária: representações sociais do cuidado à saúde bucal. Cien Saude Colet. 2006;11(1):211-8. http://dx.doi.org/10.1590/S1413-81232006000100030.
26. Elias MS, Cano MAT, Mestriner Jr W, Ferriani MGC. A importância da saúde bucal para adolescentes de diferentes extratos sociais do Município de Ribeirão Preto. Rev Lat Am Enfermagem. 2001;9(1):88-95. PMid:12046564. http://dx.doi.org/10.1590/S0104-11692001000100013.

27. Martins AMEBL, Barreto SM, Silveira MF, Santa-Rosa TTA, Pereira RD. Autopercepção da saúde bucal entre idosos brasileiros. Rev Saude Publica. 2010;44(5):912-22. PMid:20882263. http://dx.doi.org/10.1590/S003489102010005000028

28. Xie Q, Ding T, Yang G. Rehabilitation of oral function with removable dentures: still an option? J Oral Rehabil. 2015;42(3):234-42. PMid:25327636. http://dx.doi.org/10.1111/joor.12246.

29. Listl S, Behr M, Eichhammer P, Tschernig R. The psychological impact of prosthodontic treatment: a discrete response modelling approach. Clin Oral Investig. 2012;16(3):997-1006. PMid:21833483. http://dx.doi.org/10.1007/ s00784-011-0588-x.

Recebido em: Nov. 11, 2015 Aprovado em: Ago. 16, 2016 